\title{
Frozen elephant trunk: assets and liabilities of a challenging technique
}

\author{
Luca Di Marco, Daniela Votano, Alessandro Leone, Davide Pacini \\ Cardiac Surgery Unit, Cardio-Thoracic-Vascular Dept., Sant'Orsola Hospital, Bologna University, Bologna 40138, Italy. \\ Correspondence to: Dr. Luca Di Marco, Cardiac Surgery Unit, Cardio-Thoracic-Vascular Dept. Sant'Orsola Hospital, via \\ Massarenti 9, Bologna University, Bologna 40138, Italy. E-mail: ludima08@libero.it \\ How to cite this article: Di Marco L, Votano D, Leone A, Pacini D. Frozen elephant trunk: assets and liabilities of a challenging \\ technique. Vesse/ P/us 2020;4:32. http://dx.doi.org/10.20517/2574-1209.2020.23 \\ Received: 1 Jun 2020 First Decision: 15 Jun 2020 Revised: 14 Jul 2020 Accepted: 10 Oct 2020 Published: 21 Oct 2020 \\ Academic Editor: Cristiano Spadaccio Copy Editor: Cai-Hong Wang Production Editor: Jing Yu
}

\begin{abstract}
The development of the frozen elephant trunk (FET) technique for a simplified treatment of complex lesions of the thoracic aorta originated as an evolution of the classic elephant trunk technique, described for the first time by Borst et al. ${ }^{[1]}$ in 1983. Novel technologies and standardization of the surgical approach produced a progressive improvement of early and late outcomes. Most of the time and for specific indications, FET procedure allows physicians to treat lesions involving extensive portions of the thoracic aorta in one single step. Spinal cord injury remains one of the main complications of this procedure, even though spinal protection strategies have led to better results. We hereby report our opinions and recommendations based on our experience started in 2007.
\end{abstract}

Keywords: Aortic arch, acute aortic dissection, frozen elephant trunk procedure, chronic aneurysm

\section{INTRODUCTION}

Complex thoracic aortic lesions represent one of the most relevant challenges in cardiovascular surgery, often requiring more surgical and/or endovascular procedures than other diseases/injuries in the field. Since the introduction of the "Elephant Trunk" (ET) technique, described in 1983 by Borst and colleagues as a two-stage approach ${ }^{[1]}$, methods and skills have been rapidly evolving with the introduction of innovative materials and more standardized techniques ${ }^{[2-4]}$. This progression eventually led to the development of the frozen elephant trunk (FET) technique in 2003, thanks to the introduction of hybrid prostheses made up of a proximal surgical tubular graft and a distal endovascular stent-graft ${ }^{[5]}$.

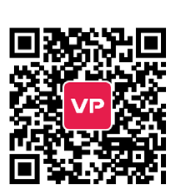




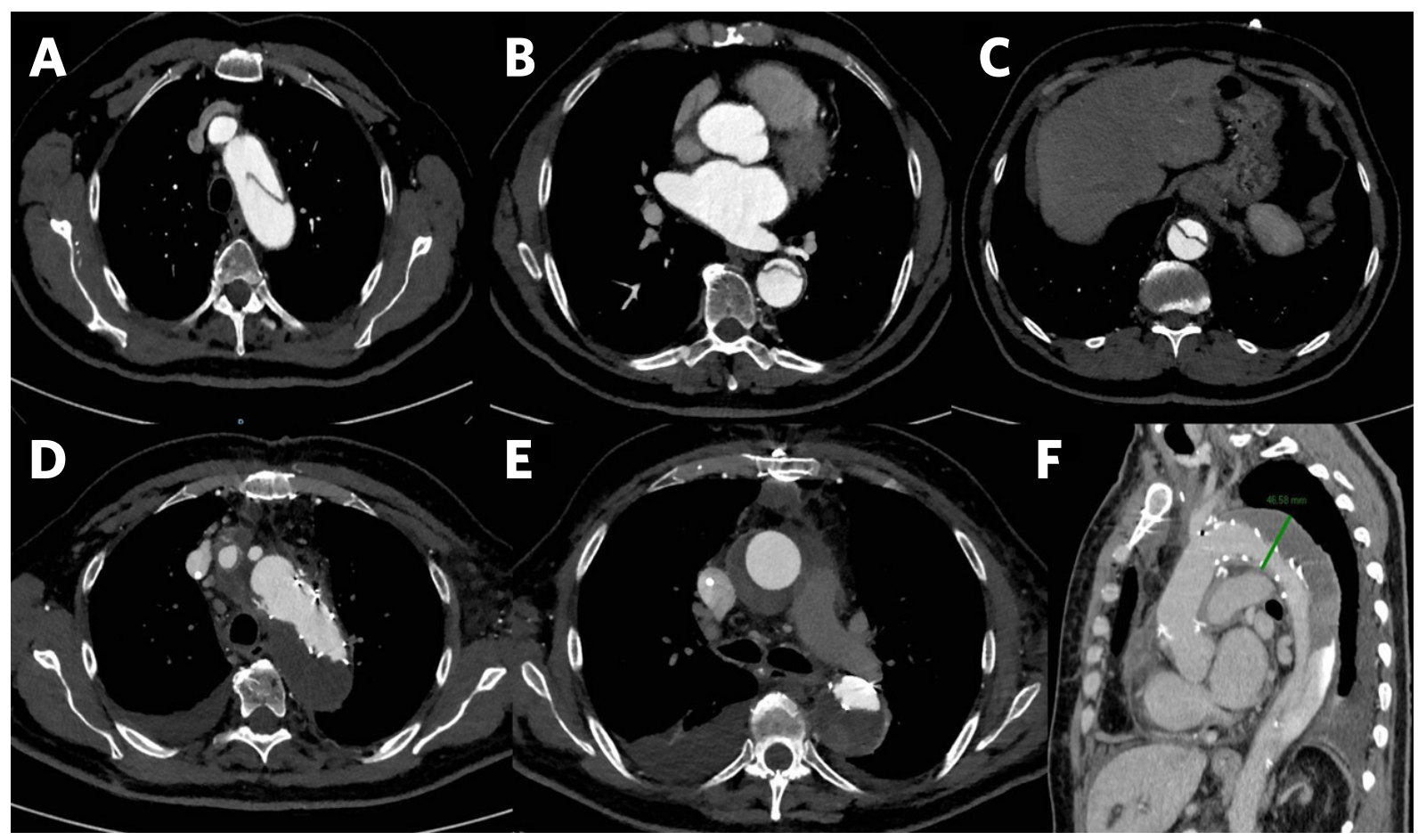

Figure 1. Frozen elephant trunk procedure with Thoraflex hybrid prosthesis in a case of acute type B aortic dissection. Pre-operative computed tomography (CT)-angiogram shows the entry tear downstream of the left subclavian artery and the dissected descending thoracic and thoracoabdominal aorta (A, B, C); two years-follow up CT-angiogram shows the complete peri-stent false lumen thrombosis (D, E, F)

The FET procedure is indicated in chronic thoracic aorta dissection, acute and chronic Stanford type B aortic dissection [Figure 1], when endovascular treatment is not feasible or contraindicated, and chronic degenerative aneurysms of the thoracic aorta and distal arch. Another indication is acute Stanford type A aortic dissection [Figure 2], especially when distal malperfusion occurs and when the intimal tear is located in the distal aortic arch or in the proximal descending thoracic aorta. A recently published position statement of the European Association for Cardio-Thoracic Surgery (EACTS) and the European Society for Vascular Surgery defines specific classes of recommendations to the aforementioned indications: in particular, class IIA is given to the cited cases of acute type A and type B aortic dissection and to aortic disease involving distal thoracic and thoraco-abdominal aorta, that is likely to require, at a later stage, either surgical or endovascular treatment. On the other hand, class IIB recommendation is given to type A aortic dissection to prevent aneurysms development in the downstream aorta ${ }^{[6]}$.

There are two available hybrid stent grafts for FET procedure in Europe with CE (Conformite Européenne) mark approval: the E-Vita Open and E-Vita Open Plus (Jotec GmbH, Hechingen, Germany) and the Thoraflex (Vascutek, Terumo, Inchinnan, Scotland, UK) [Figure 3]. These two grafts both are composed of a proximal gel-coated woven polyester tubular graft and a distal self-expanding stent graft made of polyester and nitinol ring stents. The two grafts differ from each other by the presence of three side branches for the arch vessels in the Vascutek Thoraflex prosthesis; on the other hand, the E-Vita Open Plus is a single tubular graft and the arch vessels are implanted on it with the "island" technique.

Our experience with FET procedure started in 2007 with a total of 318 procedures: 173 were carried out using the E-Vita Open hybrid prosthesis and 145 using the Thoraflex. The indications for FET procedure were chronic degenerative aortic aneurysms in 82 patients, acute type A aortic dissection in 44 patients, residual dissection in operated acute type A aortic dissection in 119 patients, chronic type A aortic 


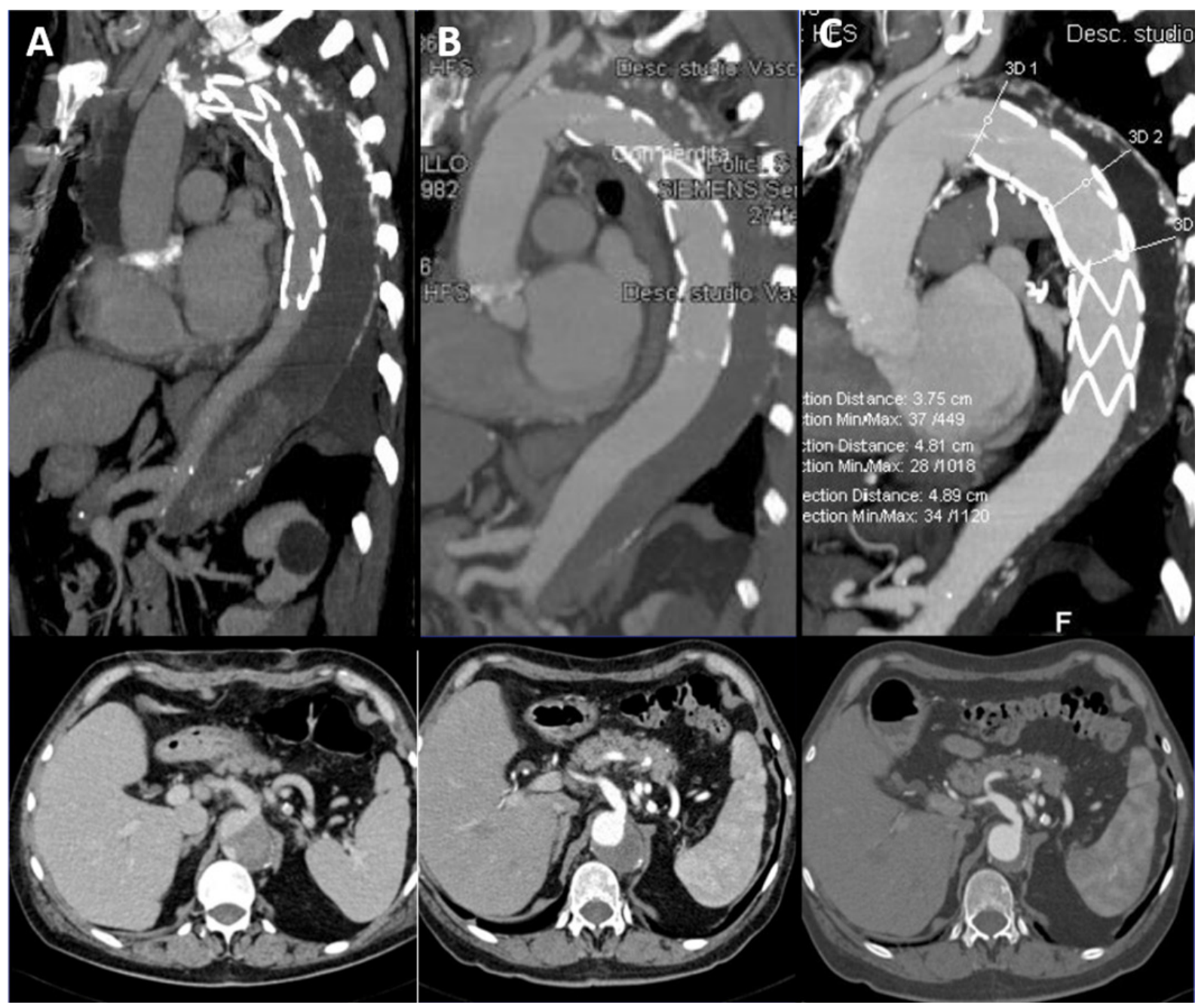

Figure 2. Frozen elephant trunk procedure with E-Vita Open plus prosthesis in a case of acute type $A$ aortic dissection with the progressive thrombosis and shrinkage of the false lumen after 1 week (A), after 3 months (B) and after 2 years with almost complete aortic remodeling (C)

dissection (not undergone surgery) in 25 patients, and acute and chronic type B aortic dissection in 13 and 35 patients, respectively. Combined procedures were $128(40.3 \%)$. With a mean follow up of 5 years, endoprosthetic extensions were performed in 85 patients (45 in E-Vita Open group and 40 in the Thoraflex group). In the vast majority of cases, the indication for extension was the incomplete thrombosis of the false lumen, whilst in very few cases, it was the inadequate distal sealing.

Some crucial recommendations we learnt from our experience are: accurate pre-operative assessment of the entire aortic anatomy, with identification of intimal tear and re-entry tears and visceral arteries origin from the true or false lumen in aortic dissections, careful evaluation of aortic diameters for appropriate graft sizing, and employment of safe and reliable organ protection and surgical strategies. In acute aortic dissection, oversizing is contraindicated since it can produce stent-induced new entry tears. Conversely, in chronic degenerative aortic aneurysms, oversizing allows an optimal distal graft sealing and it is therefore indicated.

\section{Surgical technique}

At our institution, the surgical technique for FET procedure is standardized to allow reproducibility and better outcomes. Following a full median sternotomy and administration of heparin, a stiff guide-wire is 


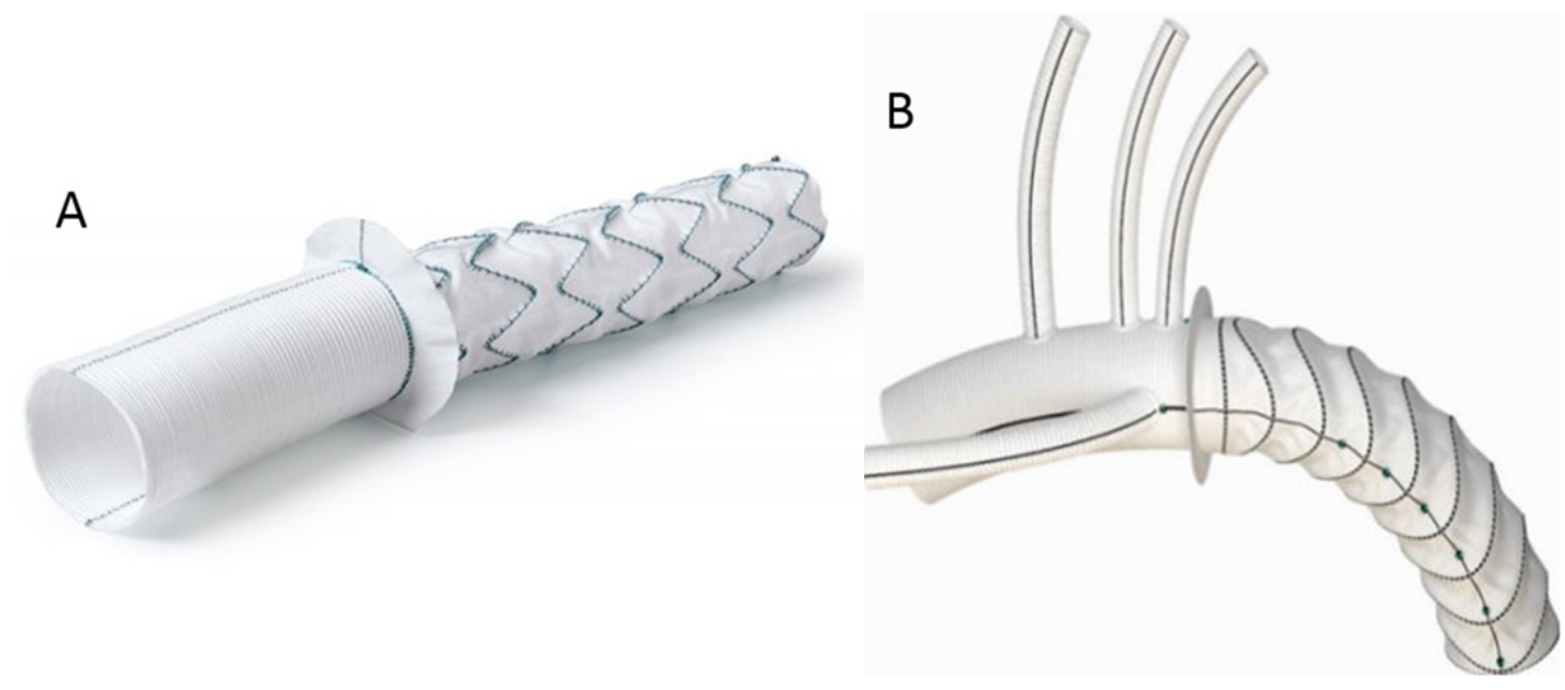

Figure 3. The E-Vita Open Plus (A) and the Thoraflex hybrid prosthesis (B)

inserted in the true lumen of the aorta up to the descending thoracic portion via the femoral artery, under transesophageal echocardiographic guidance. Cardiopulmonary bypass is then established, preferably via the right axillary artery or the right carotid artery (this can be done with interposition of an $8 \mathrm{~mm}$ Dacron graft), or directly through the brachiocephalic artery. For the venous cannulation, the chosen sites are usually the right atrium or the femoral vein, for example in case of reinterventions. A left ventricular venting cannula is inserted into the right superior pulmonary vein. Cerebral perfusion is monitored in all the cases with near infrared spectroscopy. Circulatory arrest is achieved at a nasopharyngeal temperature of $25{ }^{\circ} \mathrm{C}$. Cold crystalloid cardioplegia is administered; specifically, we use the modified Bretschneider solution (Custodiol, Koehler Chemie, Alsbach-Haenlein, Germany). Following complete resection of the arch, we use the Kazui's Antegrade Selective Cerebral Perfusion (ASCP $)^{[7]}$, specifically inserting cannulas into the left carotid and subclavian arteries, under moderate hypothermia ${ }^{[8,9]}$. The next step is the preparation of the proximal descending aorta with addition of an external Teflon strip and four internal pledgeted U-stitches. In the case of aortic dissections, we carry out a surgical obliteration of the distal stump false lumen. The prosthesis (E-Vita Open or Thoraflex hybrid device) is introduced antegradely in the descending aorta, with the guidance of the previously inserted guide-wire, and then released.

In the case of Thoraflex implantation, following release, distal anastomosis between the prosthesis collar and aorta is performed. The cardiopulmonary bypass is restarted and the systemic perfusion restored via the designated graft side branch. Thereafter, separate reimplantation of the arch vessels is performed, starting with the left subclavian artery. The proximal anastomosis is commonly carried out following the reimplantation of the left subclavian artery, with the aim to reduce cardiac ischemic time. The distal anastomosis sites are usually arch zone 3 of Ishimaru (beyond left subclavian artery), arch zone 2 (between left subclavian and left carotid artery), or more proximal sites. More proximal anastomoses are, in fact, easier to perform and carry a lower risk of left recurrent nerve damage.

In the case of E-Vita Open hybrid prosthesis implantation, following release over the guide-wire, the Dacron graft is retracted and the collar anastomosed to the distal stump. Usually the lower body is reperfused for ten minutes through the graft; thereafter the arch vessels "island" is prepared and implanted on it. Distal flow is eventually restored, and proximal anastomosis performed.

We routinely use cerebrospinal fluid (CSF) drainage as a method of spinal cord protection, positioning the lumbar catheter one day before the procedure. 
We prefer to use the Thoraflex graft when the arch vessels originate widely separately from each other or when they are severely dissected.

As already suggested by Tsagakis ${ }^{[10]}$, we routinely use angioscopy both to analyze the anatomy of the aorta before graft deployment, and to verify afterwards the correct positioning and expansion of the stent-graft.

\section{DISCUSSION}

FET technique is an elaborate procedure aimed to simplify the treatment of complex thoracic aortic lesions, which combines the classic elephant trunk technique features with the endovascular stent technology. The evolution from the classic ET to FET started at the end of the 1990s, with the "open stent-grafting technique", combining antegrade endovascular stenting with classic arch repair ${ }^{[2-4]}$. This technique was then modified by the Hannover group, with the development of a custom-made hybrid prosthesis, giving birth to the "Frozen Elephant Trunk" technique ${ }^{[5]}$. Over the last few years, hybrid prosthesis technology has evolved, until the latest introduction of branched grafts in 2012.

Outcomes of FET procedure are variably reported in the literature. In the previous EACTS 2015 position statement on the use of FET technique, analyzing data extracted from 97 focused publications available in the literature, in-hospital mortality rates of $1.8 \%$ to $17.2 \%$ have been described ${ }^{[11]}$. Similarly, Ma and colleagues showed an early mortality rate of $6.4 \%$ to $15.8 \%$ in a review article ${ }^{[12]}$.

Mortality and complication rates of FET procedure are comparable to those of classic aortic arch surgery, except for spinal cord injury (SCI), with variable rates from $0 \%$ to $21 \%$ as reported in the EACTS 2015 position statement. The most recent single-centre experience report by Shrestha and colleagues showed an in-hospital mortality of $11 \%$ (12\% in acute type A aortic dissection) and a $2 \%$ incidence of SCI ${ }^{[13]}$. Higher rates of SCI are found in patients who underwent FET procedure due to chronic aortic dissection ${ }^{[1]}$. Presumably, the mechanisms involved in the pathophysiology of SCI are coverage of descending thoracic aorta beyond T7-T8 level, longer spinal cord ischemia times, and thromboembolic events. We therefore assume that the incidence of SCI could be reduced with a shorter descending aorta coverage and spinal cord ischemia time, in addition to the validated use of CSF drainage, which we strongly recommend.

In acute and chronic aortic dissection, FET technique promotes flow restoration in the true lumen, coverage of the proximal entry and re-entry tears and thrombosis of the false lumen, either partial or complete. On this regard, the Essen group reported rates of false lumen thrombosis of $90 \%$ in acute aortic dissection and $78 \%$ in chronic dissection ${ }^{[13]}$. This induces positive aortic remodeling, with a drastic reduction of the risk of aneurysmal dilatation and rupture. Nonetheless, complete thrombosis of the false lumen could be the trigger for visceral ischemia when the visceral arteries originate from the false lumen and there are no re-entry tears located in the distal aorta. Therefore, our opinion is that FET procedure should be contraindicated in absence of re-entries in the distal thoracic, thoracoabdominal, and abdominal aorta when the visceral vessels originate from the false lumen.

In conclusion, FET technique represents a safe and effective procedure for the treatment of complex descending thoracic aortic lesions, allowing a one-stage repair and, when necessary, endovascular extension with a secure proximal landing zone. Early and late outcomes have been improving thanks to novel technologies and standardization of the surgical approach.

\section{OUR "IDEA" ABOUT FET}

Our experience with the FET technique started with the use of Jotec E-vita open prosthesis, but some years later, in 2014, we added the Thoraflex hybrid prosthesis to our "armamentarium". With the introduction of 
this new hybrid prosthesis, we expanded the indications for the FET technique also to type A and B acute aortic dissections. In this field, with the increased complexity of the cases, we began to "proximalize" the open distal anastomosis to arch zone 2 with a sensible reduction of the median visceral ischaemia time (42 min in zone 2 vs. $54 \mathrm{~min}$ in zone 3). In our opinion, this could depend on the fact that in the zone 2 group we used more frequently the Thoraflex hybrid prosthesis, allowing an earlier antegrade reperfusion through its side branch. In addition, zone 2 makes the distal anastomosis easier and faster, especially in the case of reoperations or emergency cases, so we recommend it. Furthermore, the presence of the side branches allows to perform an individual arch vessel reconstruction instead of the island technique and to restart the reperfusion in an antegrade fashion. In fact, we found that achieving an antegrade reperfusion when possible is preferable, especially in the case of chronic aneurysm. For this reason, when we use E-vita open, we create a fenestration on the vascular Dacron portion of the graft in order to antegradely reperfuse through a "Y" line for at least $10 \mathrm{~min}$ at the end of the distal anastomosis before reimplanting the left subclavian artery, which can be performed following discontinuation of the circulatory arrest, during the reperfusion-phase. In our experience, the Thoraflex hybrid prosthesis was used more frequently in arch zone 2 for acute dissections. In fact, we believe that a stent graft length of approximately $100 \mathrm{~mm}$ is sufficient to stabilize the intimal flap and to favour expansion of the true lumen in the down-stream aorta, also because a large intimal tear is often located near the left subclavian artery and can be easily excluded. The use of a shorter stent graft is also another key factor in order to reduce the rate of spinal cord injuries, especially in acute aortic dissection. This is because a shorter stent graft covers a shorter segment of the aorta and therefore fewer intercostal arteries are closed. On the other hand, to achieve a single-stage treatment in case of chronic aneurysm of the thoracic aorta, the use of a shorter stent graft, such as with Thoraflex hybrid prosthesis, is not always possible because the distance from zone 2 to the distal end of the aneurysm does not allow a perfect sealing of the stent. Therefore, in such cases, we suggest to perform the distal anastomosis in zone 3 or to use a longer stent such as the E-vita open prosthesis (130-160 mm).

\section{DECLARATION}

\section{Authors' contributions}

Substantial contribution to the concept and design of the study, data analysis and interpretation: Di Marco L, Votano D, Pacini D

Data collection, administrative, technical, and material support: Leone A

\section{Availability of data and materials}

Not applicable.

\section{Financial support and sponsorship}

None.

\section{Conflict of interest}

All authors declared that there are no conflicts of interest.

\section{Ethical approval and consent to participate}

Not applicable.

\section{Consent for publication}

Not applicable.

\section{Copyright}

(c) The Author(s) 2020. 


\section{REFERENCES}

1. Borst HG, Walterbusch G, Schaps D. Extensive aortic replacement using "elephant trunk" prosthesis. Thorac Cardiovasc Surg 1983;31:37-40.

2. Suto Y, Yasuda K, Shiiya N, Murashita T, Kawasaki M, et al. Stented elephant trunk procedure for an extensive aneurysm involving distal aortic arch and descending aorta. J Thorac Cardiovasc Surg 1996;112:1389-90.

3. Miyamoto S, Hadama T, Anai H, Sako H, Shigemitsu O. Stented elephant trunk method for multiple thoracic aneurysm. Ann Thorac Surg 2001;71:705-7.

4. Kato M, Ohnishi K, Kaneko M, Ueda T, Kishi D, et al. New graft-implanting method for thoracic aortic aneurysm or dissection with a stented graft. Circulation 1996;94:II188-93.

5. Karck M, Chavan A, Hagl C, Friedrich H, Galanski M, et al. The frozen elephant trunk technique: a new treatment for thoracic aortic aneurysms. J Thorac Cardiovasc Surg 2003;125:1550-3.

6. Czerny M, Schmidli J, Adler S, van den Berg JC, Bertoglio L, et al. Current options and recommendations for the treatment of thoracic aortic pathologies involving the aortic arch: an expert consensus document of the European Association for Cardio-Thoracic surgery (EACTS) and the European Society for Vascular Surgery (ESVS). Eur J Cardiothorac Surg 2019;55:133-62.

7. Kazui T, Inoue N, Yamada O, Komatsu S. Selective cerebral perfusion during operation for aneurysms of the aortic arch: a reassessment. Ann Thorac Surg 1992;53:109-14.

8. Pacini D, Leone A, Di Marco L, Marsilli D, Sobaih F, et al. Antegrade selective cerebral perfusion in thoracic aorta surgery: safety of moderate hypothermia. Eur J Cardiothorac Surg 2007;31:618-22.

9. Di Bartolomeo R, Di Marco L, Armaro A, Marsilli D, Leone A, et al. Treatment of complex disease of the thoracic aorta: the frozen elephant trunk technique with the E-vita open prosthesis. Eur J Cardiothorac Surg 2009;35:671-5.

10. Tsagakis K. Angioscopy as a supplement to frozen elephant trunk treatment. Ann Cardiothorac Surg 2013;2:653-5.

11. Shrestha M, Bachet J, Bavaria J, Carrel TP, De Paulis R, et al. Current status and recommendations for use of the frozen elephant trunk technique: a position paper by the Vascular Domain of EACTS. Eur J Cardiothorac Surg 2015;47:759-69.

12. Ma WG, Zheng J, Sun LZ, Elefteriades JA. Open stented grafts for frozen elephant trunk technique: technical aspects and current outcomes. Aorta (Stamford) 2015;3:122-35.

13. Shrestha M, Martens A, Kaufeld T, Beckmann E, Bertele S, et al. Single-centre experience with the frozen elephant trunk technique in 251 patients over 15 years. Eur J Cardiothorac Surg 2017;52:858-66. 